\title{
Comparative Study of the Effects of Thermal and Photochemical Accelerated Oxidations on Quality of "Green Type" and "Black Type" French Olive Oils
}

\author{
Jérôme Plard, Yveline Le Dréau, Catherine Rébufa, Nathalie Dupuy
}

Aix Marseille Université, CNRS, IRD, Avignon Université, IMBE, UMR 7263, équipe BEC, case 451, Avenue Escadrille Normandie Niémen, 13397 Marseille cedex 20, France

Email: nathalie.dupuy@univ-amu.fr

How to cite this paper: Plard, J., Le Dréau, Y., Rébufa, C. and Dupuy, N. (2016) Comparative Study of the Effects of Thermal and Photochemical Accelerated Oxidations on Quality of "Green Type" and "Black Type" French Olive Oils. American Journal of Analytical Chemistry, 7, 890-907. http://dx.doi.org/10.4236/ajac.2016.712076

Received: October 6, 2016

Accepted: December 16, 2016

Published: December 19, 2016

Copyright $\odot 2016$ by authors and Scientific Research Publishing Inc. This work is licensed under the Creative Commons Attribution International License (CC BY 4.0).

http://creativecommons.org/licenses/by/4.0 1

(c) (i)

Open Access

\begin{abstract}
Oxidative stability of two commercial olive oils of different specificity (green type and black type) has been studied during thermal and photochemical accelerated processes through the evolution of quality indices. It might help to assure a good utilisation of olive oil. In most of works described in literature, they are measured individually. In this study, a Principal Component Analysis (PCA) has been performed to emphasize their variation and describe in concise way the quality and the safety of extra-virgin olive oil after two oxidative stresses. No difference had been detected between both type oils when they are heated. Peroxides, aldehydes and conjugated dienes and trienes were formed but rapidly degraded into final oxidation compounds, mainly acid compounds. During the photochemical process, similar changes occurred slower and the green type oil had shown better stability because of its higher phenolic content. The fatty acids had been more impacted (higher disappearance of monounsaturated fatty acids (MUFA) and polyunsaturated fatty acids (PUFA)) when the oils were heated than when irradiated. Saturated fatty acids (SFA), MUFA and PUFA were the most relevant indicators to characterize non-oxidized oils and PV characterized the early stage of oil oxidation.
\end{abstract}

\section{Keywords}

Olive Oil, Photo-Oxidation, Thermal Oxidation, Quality Control Parameters, Fatty Acids, Phenolic Compounds

\section{Introduction}

Extra-virgin olive oil (EVOO) is considered to be the best olive oil for its organoleptic 
characteristics, its stability and its chemical composition because it contains important nutritional elements (fatty acids, vitamins, etc.). The other categories of olive oil have less taste and aroma. Thus, to estimate the chemical oil quality, the International Olive Council (IOC) and the International Organisation for Standardisation (ISO) recommend to measure quality indices such as free acidity (FA) [1], peroxide value (PV) [2], $\mathrm{K}_{232}$ and $\mathrm{K}_{270}$ indices [3], and anisidine value (AV) [4]. Lower and upper values of these indices have been established by the Regulations of the European Union Commission [5]. So different standard: 1) fixe the physico-chemical, 2) distinguishing quality and purity criteria of each designation (grade) of olive oil, 3) harmonize national and international laws relating to, in particular the marketing of olive oil, 4) help protect consumers and prevent fraudulent practices. In addition, the fatty acids [6] and the phenolic compounds [7] are often quantified because of their active participation to the resistance of olive oil deterioration. Numerous studies showed that drastic storage and using conditions of EVOO (long-time light exposure and during cooking) induced lipid oxidation that degraded the organoleptic properties of oil. A series of reactions such as hydrolysis, oxidation, polymerisation, isomerisation and cyclisation (at $180^{\circ} \mathrm{C}$ or above) can occur and lead to the formation of peroxides and hydroperoxides transformed later into secondary oxidative products such as hydrocarbons, alcohols, aldehydes, ketones and carboxylic acids [8] suspected to be hazardous to the human health. The preservation of the specific taste, odour and nutritive properties is a major challenge for both consumers and food industry which develops patents for olive oil preservation by freezing or deep-freezing [9] or confines olive oil in biopolymers envelope to accurate shaping of individual olive oil, portions for immediate use in cooking [10]. The review of Santos et al. [11] focused on studies dealing with heating processes (laboratory simulations or cooking conditions) and resumed the chemical parameters used to follow the olive oil quality attributes. In previous papers, all quality parameters are rarely measured simultaneously to follow the EVOO degradation under UV irradiation or cooking conditions. It appears that their response to an oxidative stress is different and the correlation between them is not clearly highlighted. FA is the commonly used quality index and it has been observed that it hardly varies during the heating of the oil to $180^{\circ} \mathrm{C}$. However, when foods are fried in oil, FA slightly increases proportionally to the frying time [12] [13]. Cheikhousman et al. [14] and Daskalaki et al. [15] reported that $\mathrm{PV}$ increased when virgin olive oils (VOO) was heated $3 \mathrm{~h}$ at $170^{\circ} \mathrm{C}$ or $1 \mathrm{~h}$ at $180^{\circ} \mathrm{C}$, before decreases. This increase is confirmed after frying processes in presence of food in EVOO or in VOO [11] [12]. At low temperature $\left(\leq 100^{\circ} \mathrm{C}\right)$, a latency period is observed [16] [17] [18]. Similar increase of AV is found for olive oils of different qualities [13]. Oils heated $3 \mathrm{~h}$ at $180^{\circ} \mathrm{C}$ show a faster degradation of the more unsaturated fatty acids [15]. The heat treatment $\left(4 \mathrm{~h}\right.$ at $\left.180^{\circ} \mathrm{C}\right)$ caused a reduction in the fatty acid content which mainly affected the PUFA fraction (about 13\%) and a slight reduction in the MUFA content was noticed: oleic acid content reduction was lower than $1 \%$, despite being the most abundant FA [19]. Gómez-Alonso et al. [20] reported that linoleic acid

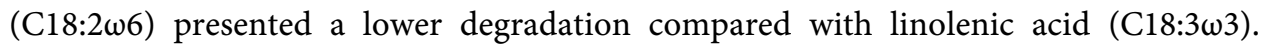
Bešter et al. [21] confirmed a progressive decrease in linolenic acid (C18:3 $\omega 3)$ and li- 


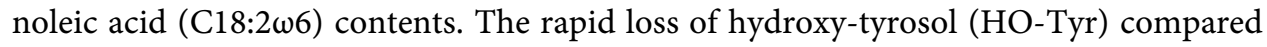
with fatty acids translated its effective antioxidant activity in protecting lipids against oxidation. The antioxidant efficiency of tyrosol (Tyr) is relatively low. It is less degraded upon frying, but nevertheless shows a linear rate of degradation during time [15] [21] [22].

Impact of light on olive oils has also aroused the interest of some researchers. Under an artificial light at high intensity, PV has quickly overstepped the European regulation limit for EVOO ( $\left.\leq 20 \mathrm{meq} \mathrm{O}_{2} \cdot \mathrm{kg}^{-1}\right)$ and the ortho-diphenols quantities have decreased [23]. Poulli, Mousdis and Georgiou [24] (2009) have studied the impact of UV irradiation coupled to the heat (between $20^{\circ} \mathrm{C}$ and $80^{\circ} \mathrm{C}$ ). They have shown that PV increases greater than $\mathrm{AV}$ for a same UV irradiation intensity when temperature was high. PV of oil sample studied by Rahmani and Csallany [25] increased significantly (up to 320 meq $\mathrm{O}_{2} \cdot \mathrm{kg}^{-1}$ ) after $120 \mathrm{~h}$ under a high light irradiation at $2^{\circ} \mathrm{C}$. However, at $40^{\circ} \mathrm{C}, \mathrm{PV}$ decreases after having reached a maximum. Moreover, $\mathrm{PV}, \mathrm{K}_{232}$ and $\mathrm{K}_{270}$ levels of olive oils were also significantly increased by not intensive lighting storage conditions (fluorescent lamps, 482 lux, 12 months, room temperature) [26]. When olive oil samples of are exposed to the UV lamp during 12 days, the rates of phenols decrease during the first five days while the percentage of unsaturated fatty acid decreased only from the fifth day [27]. It highlights the antioxidant activity of phenols. Furthermore, the works of Nieto et al. [28] done with an UV lamp immersed into a temperature controlled photo-reactor containing $1 \mathrm{~L}$ of olive oil shows weak variations of PV, FA, $\mathrm{K}_{232}$ and $\mathrm{K}_{270}$, thus demonstrating that volume is also an important factor influencing the oxidation of lipids. Studies on EVOO samples transferred in dark glass bottles (headspace $0.5 \%$ ) and stored without heating during 24 months ${ }^{\text {cat3 }}$ showed that FA did not change during storage while extinction coefficient and PV were gradually increased and reductions up to $25 \%$ and $31 \%$ in the phenolic content (particularly the most polar secoiridoids) were recorded after 18 and 24 months [29].

Most of these studies have focused on the effect of either heat or light on the lipid oxidation in olive oils through measurements of some quality indices but results were difficult to compare because of the variability of the initial oil composition and the ageing processes. With this in mind, this work aimed to investigate the influence of two accelerated processes (heating at $180^{\circ} \mathrm{C}$-usual cooking temperature, or exposition at UV irradiation) on the main quality parameters and SFA, MUFA, PUFA, Tyr and HO-Tyr contents obtained every $30 \mathrm{~min}$ for $210 \mathrm{~min}$ for two commercial virgin olive oils from the same cultivar with different specificity: one "green type" EVOO and one "black type" VOO. The knowledge of the evolution of these parameters all together should help to assure a good utilisation of olive oil. As many parameters were measured and expressed with their own units, it is not easy to compare them. Thus a Principal Component Analysis (PCA) is performed to emphasize their variation and describe in concise way the quality and the safety of extra-virgin olive oil after two oxidative stresses. Furthermore, PCA plots allowed highlighting the quality parameters essential to characterize the oil type and the different oxidation stages of oil. 


\section{Materials and Methods}

\subsection{Samples}

Two commercial virgin olive oils coming from the same mill (Auriol, France) were used throughout the oxidative studies to avoid the bias due to the parameters influencing the chemical composition other than the olive fruit maturity. These oils were obtained from olive fruits of the same cultivar (Aglandau, the most widespread in the Aix-en-Provence region) harvested during the crop season 2009-2010 at different degree of ripeness, resulting in oil composition differences. The first, an EVOO, named "green type" (G) oil, was obtained immediately after harvesting of olives before or during colour change and the second, a VOO, named "black type" (B) was obtained from olives harvested at maturity but crushed after a controlled fermentation of a few days. Oils of this type have lost the characteristics of fresh fruits but present, nowadays, a specific and well-identified taste and enjoy of a renewed interest from consumers wanting oils with constant gustative qualities, although they do not always meet the criteria of the EVOO [30] [31]. The choice of black type oil has been made to increase knowledge on this oil category by comparison to green type oil.

\subsection{Oxidative Processes}

Five grams of oils were placed in drastic conditions (with bubbling oxygen at 75 $\mathrm{mL} \cdot \mathrm{min}^{-1}$ ) to accelerate oxidation. For every condition and for every specific period (30, $60,90,120,150,180$ and 210 minutes), the process was performed 5 times, then, the oxidized oils were mixed together to obtain a sufficient volume for chemical analyses.

\subsubsection{Thermal Process}

Oil sample was heated at $180^{\circ} \mathrm{C} \pm 2^{\circ} \mathrm{C}$ under stirring in a glass tube, placed in a silicone oil bath. Every time period was counted down after $10 \mathrm{~min}$, in order to take into account the required time so that the oil reaches $180^{\circ} \mathrm{C}$.

\subsubsection{Photochemical Process}

Oil sample placed in a glass tube was kept under stirring. A mercury arc lamp (ORIEL, model 6286, 250 watts), equipped with a water filter and an ozone trap, placed at $5 \mathrm{~cm}$ from the glass tube, was used to illuminate the sample horizontally in UV domain between 200 and $2500 \mathrm{~nm}$. A concave mirror placed at $5 \mathrm{~cm}$ on the other side of the glass tube was used to refocus UV radiations on sample.

\subsubsection{Samples Designation}

Oil samples thermally $(\mathrm{T})$ and photochemically $(\mathrm{P})$ oxidized under different time period were noted T0, T30, .., T210 and P30, ..., P210. "FO" designed the fresh oil while "T0" designed the oil heated during $10 \mathrm{~min}$, time to reach $180^{\circ} \mathrm{C}$. For the samples designation on PCA plots, the first letter indicates oil type ( $G$ or B), the second, the oxidative process ( $\mathrm{T}$ or $\mathrm{P}$ ) and the number the time.

Experiments were realized in triplicates to obtain the mean values \pm standard deviation of their chemical indices ( $\mathrm{PV}, \mathrm{AV}, \mathrm{FA}, \mathrm{K}_{232}$ and $\mathrm{K}_{270}$ ), their fatty acid composition 
and their Tyr and HO-Tyr contents.

\subsection{Quality Indices}

Peroxide value (PV), expressed as milliequivalents of active oxygen per kilogram of oil (meq $\mathrm{O}_{2} \cdot \mathrm{kg}^{-1}$ ), was determined according to ISO standard method 3960 [2] using chloroform instead of isooctane, as Talpur et al. [32] recommended, to solubilise correctly the highly oxidized oils. The anisidine value (AV) was measured according to ISO standard method 6885 [4]. For more efficiently quantify oxidation, TOTOX [24]. Free acidity (FA) expressed as percentage of oleic acid was obtained according to ISO standard method 660 [1]. UV absorption characteristics at 232 and $270 \mathrm{~nm}$ were carried out following analytical method described in the IOC method [3] (IOC, 2010). Cyclohexane was used instead of isooctane, to correctly solubilise the highly oxidized oils. UV-visible spectroscopy analyses for the determination of quality indices $K_{232}, K_{270}$ and AV, were carried out with a spectrometer Thermo Electron Corporation Evolution 300 (Boston, Massachusetts, USA). Instrument control and initial data processing were performed using VISION PRO software (v 1.02).

\subsection{Fatty Acids Composition}

Determination of fatty acids composition was based on the analysis by GC of fatty acid methyl esters (FAMEs) prepared according to IOC method [6]. A solution of nonadecanoate methyl ester $\left(\mathrm{C}_{19}, 2 \mathrm{mg} \cdot \mathrm{mL}^{-1}\right.$ in isooctane) was used as internal standard to calculate each fatty acid content as mg.eq $\mathrm{C}_{19} \cdot \mathrm{g}^{-1}$ oil. A weighed quantity of olive oil sample $(0.120 \pm 0.001 \mathrm{~g})$ introduced in a centrifuge tube, with $2 \mathrm{~mL}$ of solution of the internal standard was transmethylated using $0.2 \mathrm{~mL}$ of cold methanolic solution of $\mathrm{KOH}(2 \mathrm{M})$. The mixture was stirred 2 min with agitator for test tubes, diluted with 1 $\mathrm{mL}$ of isooctane and centrifugated at $3900 \mathrm{rpm}$ for $5 \mathrm{~min}$. An aliquot of the solvent phase was taken out for injection. The fatty acid methyl esters (FAMEs) were analysed ( $1 \mu \mathrm{L}$ injected) on a GC Agilent 7890 A GC System (Santa Clara, California', USA) equipped with a split/split-less injector $\left(250^{\circ} \mathrm{C}\right)$, a flame ionization detector $\left(250^{\circ} \mathrm{C}\right.$, hydrogen: $35 \mathrm{~mL} \cdot \mathrm{min}^{-1}$, air: $\left.350 \mathrm{~mL} \cdot \mathrm{min}^{-1}\right)$ a Supelcowax 10 column $(60 \mathrm{~m}$ length $\times 0.25$ $\mathrm{mm}$ internal diameter, film thickness $0.25 \mu \mathrm{m}$; Supelco, Bellefonte, Pennsylvania, USA). The inlet flow of the hydrogen as carrier gas was $1 \mathrm{~mL} \cdot \mathrm{min}^{-1}$ with split ratio $1: 70$. Oven temperature program was as follows: $20 \mathrm{~min}$ at $210^{\circ} \mathrm{C}$, from $210^{\circ} \mathrm{C}$ to $245^{\circ} \mathrm{C}$ at $6^{\circ} \mathrm{C} \cdot \mathrm{min}^{-1}, 17 \mathrm{~min}$ at $245^{\circ} \mathrm{C}$. Instrument control and data processing were performed using Chemstation software (Rev.B.04.01). FAMEs were identified by comparing their retention time to standard samples. Analyzed fatty acids were classified into three categories: SFA, containing C16:0, C17:0, C18:0, C20:0, C22:0 and C24:0, MUFA, containing $\mathrm{C} 16: 1 \omega 9, \mathrm{C} 16: 1 \omega 7, \mathrm{C} 17: 1 \omega 8, \mathrm{C} 18: 1 \omega 9, \mathrm{C} 18: 1 \omega 7$ and $\mathrm{C} 20: 1 \omega 9$, and PUFA, containing $\mathrm{C} 18: 2 \omega 6$ and $\mathrm{C} 18: 3 \omega 3$. The concentration $\left(C_{\mathrm{i}}\right)$ of the methyl ester of the fatty acid is calculated from the Equation (1):

$$
C_{i}=\frac{A_{i} \times C_{e}}{A_{e}} \times \frac{k_{i}}{k_{e}}
$$


where $C_{e}$ is the concentration of the internal standard $\left(2 \mathrm{mg} \cdot \mathrm{mL}^{-1}\right), A_{i}$, the peak area of the methyl ester and $A_{e}$, the peak area of the internal standard. The response factors $k_{i}$ are considered identical for all the compounds and so the ratio $k_{\mathrm{i}} / k_{\mathrm{e}}$ is considered equal to 1 . Final results, calculated on the basis of the analyzed oil weight, were expressed in mg.eq $\mathrm{C}_{19} \cdot \mathrm{g}^{-1}$ oil.

\subsection{Phenolic Components}

Determination of phenolic content was based on the analysis by HPLC of phenolic extracts of samples. A solution of syringic acid $\left(0.015 \mathrm{mg} \cdot \mathrm{mL}^{-1}\right.$ methanol/water $(80 / 20$, $\mathrm{v} / \mathrm{v})$ ) was used as internal standard. A solution of Tyr $\left(0.030 \mathrm{mg} \cdot \mathrm{mL}^{-1}\right.$ in methanol/water) was used to calculate the mean relative response factor of Tyr versus syringic acid $\left(\mathrm{RRF}_{\text {Syr/Tyr }}\right)$ in order to express Tyr and HO-Tyr contents (the two most commonly studied compounds as indicators of polyphenols content) as mg.eq Tyr $\mathrm{kg}^{-1}$ oil as described in the IOC testing methods [7]. A weighed quantity of olive oil sample (2.0 \pm $0.001 \mathrm{~g}$ ) was introduced in a centrifuge tube, and $1 \mathrm{~mL}$ of solution of syringic acid and 1 $\mathrm{mL}$ of methanol/water $(80 / 20, \mathrm{v} / \mathrm{v})$ was added. The mixture was stirred $5 \mathrm{~min}$ with agitator for test tubes and centrifugated at $3900 \mathrm{rpm}$ for $12 \mathrm{~min}$. The solvent phase was taken out and oil residue was extracted a second time with $2 \mathrm{~mL}$ of methanol/water. The both solvent phases were mixed and evaporated in a rotary evaporator at $40^{\circ} \mathrm{C}$ under vacuum. The dry residue was dissolved in $1 \mathrm{~mL}$ of methanol and were analysed (20 $\mu \mathrm{L}$ injected) on a LC Agilent 1200 series system equipped with an auto-sampler, a quaternary pump, column heater module $\left(25^{\circ} \mathrm{C}\right)$, and a photodiode array detector operated with Chemstation software. Two coupled chromolith RP18e $(100 \times 4.6 \mathrm{~mm})$ (Merk, Darmstadt, Germany) columns preceded by a guard column (RP18e, $5 \times 4.6 \mathrm{~mm}$ ) were used. Separation was achieved by elution gradient $\left(1 \mathrm{~mL} \cdot \mathrm{min}^{-1}\right)$ using an initial composition of $96 \%$ water with $0.2 \%$ acetic acid (A) and $4 \%$ methanol/acetonitrile (50/50, v/v) (B). The concentration of B was increased to $50 \%$ in $40 \mathrm{~min}$, and then it was raised to $60 \%$ in $5 \mathrm{~min}$ and to $100 \%$ in $15 \mathrm{~min}$. This concentration was maintained for $10 \mathrm{~min}$ before decreasing to $4 \%$ (initial composition) in $2 \mathrm{~min}$. This composition was maintained for $10 \mathrm{~min}$. Detection was performed at $280 \mathrm{~nm}$. Tyr and HO-Tyr peaks were identified by comparing their retention time to standard ones, and on the base of literature data [21] [33]. Tyr and HO-Tyr contents were calculated from Equation (2):

$$
\text { Content }_{\text {phenol }}=\frac{A_{i} \times W_{\text {Syr }} \times 1000 \times R^{2} F_{\text {Syr } / \text { Yyr }}}{A_{\text {Syr }} \times W_{\text {oil }}}
$$

where $A_{i}$ is the peak area of the compound to quantify, $A_{\mathrm{syr}}$ is the peak area of syringic acid, $W_{\text {syr }}$ is the weight $(\mathrm{mg})$ of syringic acid in $1 \mathrm{ml}$ of internal standard solution added to sample, $W_{\text {oil }}$ is the oil weight $(\mathrm{g}), 1000$ a multiplier coefficient to express the final result in $\mathrm{mg} \cdot \mathrm{kg}^{-1}$ oil. $R R F_{\text {Syr } / \mathrm{Tyr}}$ was calculated according to Equation (3) form a calibration range injected before each series of samples.

$$
R R F_{\mathrm{Syr} / \mathrm{Tyr}}=\frac{A_{\mathrm{syr}}}{C_{\mathrm{Syr}}} \times \frac{C_{\mathrm{Tyr}}}{A_{\mathrm{Tyr}}}
$$


where $A_{\mathrm{syr}}$ is the peak area of syringic acid, $A_{\mathrm{Tyr}}$ is the peak area of Tyr, $C_{\mathrm{Syr}}$ is the concentration of acid syringic standard solution and $C_{\mathrm{Tyr}}$ is the concentration of Tyr standard solution.

\subsection{Reagents}

All chemicals and reagents used were of analytical grade. Chloroform ( $\geq 99 \%)$, acetic acid glacial ( $\geq 99.5 \%$ ), diethyl ether ( $\geq 99.8 \%$ ) and cyclohexane ( $\geq 99.8 \%)$ were obtained from Carlo Erba Reactifs SDS (Val de Reuil, France), isooctane ( $\geq 99 \%)$ from Sigma-Aldrich (Steichein, Germany). Acetonitrile and methanol (HPLC grade, $\geq 99.9 \%$ ) were purchased from Sigma Aldrich (Steichein, Germany). Milli-Q ultrapure water was purified in the laboratory by an ultrapure water purification system (Millipore-Merck KGaA, Darmstadt, Germany). Sodium thiosulfate $(\geq 99 \%)$ and potassium iodide $(\geq 99 \%)$ were purchased from Sigma-Aldrich, sodium carbonate ( $\geq 99.5 \%)$ from Carlo Erba Reactifs SDS, potassium hydrogenophtalate $(\geq 99.8 \%)$ from Prolabo (Fontenay-sous-Bois, France) and p-anisidine ( $\geq 99 \%$ ) from Alfa Aesar (Johnson Matthey Company, Karlsruhe, Germany). Nonadecanoate methyl ester ( $\geq 99 \%)$, syringic acid $(\geq 95 \%)$ and tyrosol ( $\geq 98 \%)$ were purchased from Sigma-Aldrich.

\subsection{Statistic Analysis}

Via the ANOVA approach, the experimental data obtained for both green and black type oils were analysed by the F-test to determine the variances equivalence of two data sets differing in the oil types or oxidative processes. The F-test generates a p-value. If p $>0.05$ (at 95\% confidence) then the null hypothesis that the variances are equivalent cannot be rejected, otherwise, the null hypothesis cannot be accepted. According to the measured parameter, when it can be safely accepted that the variances of the two sets are equivalent, the two oils are considered having the same behaviour.

\subsection{Principal Component Analysis}

Principal component analysis (PCA) is a method of data analysis which converts many correlated variables in many uncorrelated variables named principal components (PC) [34]. It reduces information and explains variability of samples with a minimum of information. PCA was performed on all the mean values of the chemical analyses (PV, AV, TOTOX, FA, $\mathrm{K}_{232}, \mathrm{~K}_{270}$, SFA, MUFA, PUFA, Tyr and HO-Tyr contents) without excluding samples, by using full cross validation and dividing variable values by standard deviation.

\subsection{Software}

ANOVA and PCA were performed using the Unscrambler software version 10.3 from CAMO (Computer Aided Modelling, Trondheim, Norway).

\section{Results and Discussion}

\subsection{Fresh Oils}

Table 1 shows the chemical indices of the two fresh oils before treatment. The ANOVA 
Table 1. Chemical quality parameters of green type oil (G) and black type oil (B) before treatment.

\begin{tabular}{|c|c|c|c|c|}
\hline & \multirow{2}{*}{ G } & \multirow{2}{*}{ B } & \multicolumn{2}{|c|}{ European regulation limit (EEC, 2013) } \\
\hline & & & EVOO & Voo \\
\hline $\mathrm{PV}\left(\right.$ meq $\left.\mathrm{O}_{2} \cdot \mathrm{kg}^{-1}\right)$ & $15 \pm 1^{\mathrm{a}}$ & $21 \pm 2^{\mathrm{b}}$ & $\leq 20$ & $\leq 20$ \\
\hline AV & $9 \pm 1^{\mathrm{a}}$ & $4 \pm 0^{\mathrm{b}}$ & & \\
\hline TOTOX & $40 \pm 3^{\mathrm{a}}$ & $48 \pm 3^{\mathrm{a}}$ & & \\
\hline FA $(\% \mathrm{C} 18: 1)$ & $0.3 \pm 0.02^{\mathrm{a}}$ & $0.9 \pm 0.1^{\mathrm{b}}$ & $\leq 0.8$ & $\leq 2$ \\
\hline $\mathrm{K}_{232}$ & $2.49 \pm 0.16^{\mathrm{a}}$ & $2.80 \pm 0.17^{\mathrm{b}}$ & $\leq 2.50$ & $\leq 2.60$ \\
\hline $\mathrm{K}_{270}$ & $0.17 \pm 0.02^{\mathrm{a}}$ & $0.17 \pm 0.02^{\mathrm{a}}$ & $\leq 0.22$ & $\leq 0.25$ \\
\hline $\mathrm{SFA}\left(\mathrm{mg} \cdot \mathrm{eq} \mathrm{C}_{19} \cdot \mathrm{g}^{-1}\right)$ & $148 \pm 13^{\mathrm{a}}$ & $148 \pm 13^{\mathrm{a}}$ & & \\
\hline MUFA(mg.eq $\left.\mathrm{C}_{19} \cdot \mathrm{g}^{-1}\right)$ & $720 \pm 36^{\mathrm{a}}$ & $693 \pm 35^{\mathrm{a}}$ & & \\
\hline PUFA(mg.eq $\left.\mathrm{C}_{19} \cdot \mathrm{g}^{-1}\right)$ & $96 \pm 5^{\mathrm{a}}$ & $101 \pm 6^{\mathrm{a}}$ & & \\
\hline Tyr (mg.eq Tyr.kg $\left.{ }^{-1}\right)$ & $80 \pm 4^{\mathrm{a}}$ & $24 \pm 1^{\mathrm{b}}$ & & \\
\hline HO-Tyr (mg.eq Tyr.kg ${ }^{-1}$ ) & $52 \pm 2^{\mathrm{a}}$ & $11 \pm 0.4^{\mathrm{b}}$ & & \\
\hline
\end{tabular}

Values are the means \pm standard deviation of 5 replicate analyses. EVOO, extra-virgin olive oil; PV, peroxide value; $\mathrm{AV}$, p-anisidine value; TOTOX, total oxidation value; FA, free acidity; C18:1, oleic acid; $\mathrm{K}_{232}$, spectroscopic absorption at $232 \mathrm{~nm} ; \mathrm{K}_{270}$, spectroscopic absorption at $270 \mathrm{~nm}$; SFA, saturated fatty acids; $\mathrm{C}_{19}$, nonadecanoic acid; MUFA, mono unsaturated fatty acids; PUFA, poly unsaturated fatty acids; Tyr, tyrosol; HO-Tyr, hydroxyl-tyrosol. Values with the same letter in the same line are not significantly different at $95 \%$ confidence.

results, given throughout the discussion, are transcribed by a letter in the table: values with the same letter in the same line are not significantly different ( $\mathrm{P}$-value $>0.05)$. The green type oil has values of $\mathrm{PV}$ and FA lower than the maximum values indicated for "EVOO" category by the EEC Regulations [5] i.e. respectively $20 \mathrm{meq} \mathrm{O}_{2} \cdot \mathrm{kg}^{-1}$ and $0.8 \%$. The black type oil is classified in the "VOO" category especially because of its higher FA value ( $\leq 2 \%[5])$ and presents a low content of Tyr and HO-Tyr. This is a consequence of the maturity and the fermentation of olives which lead to degradation processes. Furthermore, black type oil has a lower AV value because its oxidation has continued until the formation of acid forms without the protection of the phenolic antioxidants. This may explain its high value of FA. $\mathrm{K}_{232}$ and $\mathrm{K}_{270}$ and SFA, MUFA and PUFA contents are almost identical for the two oils because they are made with the olive cultivar.

\subsection{Oxidized Oils}

\subsubsection{Chemical Indices}

The samples are treated by thermal or photochemical process, and results obtained from chemical analyses are compared to evaluate the impact of the initial oil quality and aging process. Figure 1 presents the evolution of PV, AV and TOTOX, for green and black oil types submitted to a thermal-oxidation at $180^{\circ} \mathrm{C}$ (Figures 1 (a)-(c)) and to a photochemical treatment (Figures $1(\mathrm{~d})$-(f)). The letters transcribed ANOVA results: if they are different for the same treatment, the behaviour of both oils is significantly different; if they are different for the same oil for the two treatments, the influence of 

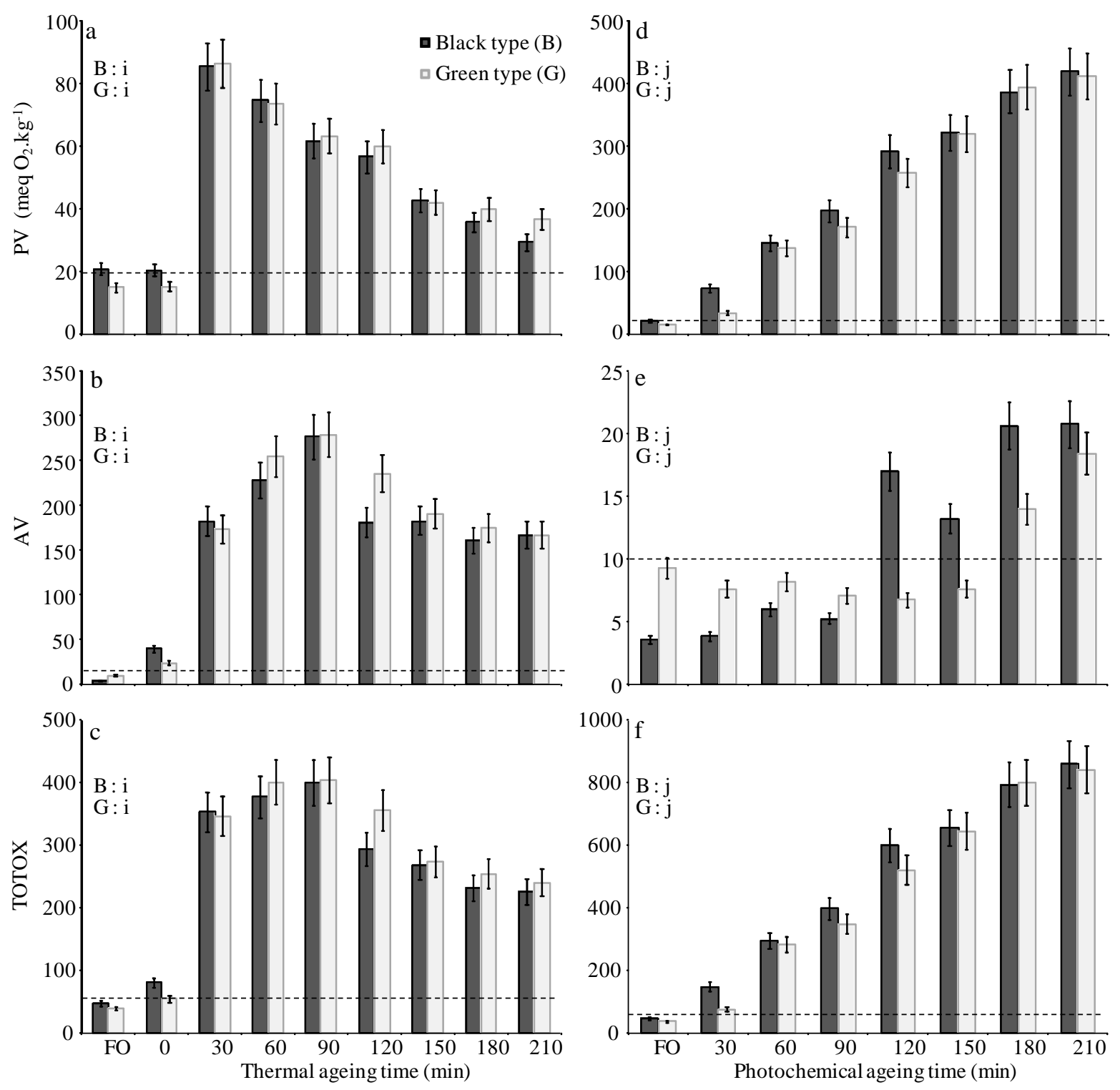

Figure 1. Evolution of (a, d) peroxide value (PV), (b, e) anisidine value (AV) and (c, f) TOTOX according thermal and photochemical ageing time. (Limit fixed for PV by the European regulation for EVOO (EEC, 2013), for AV according to different authors (Naz et al., 2005; Casal et al., 2010) and for TOTOX by calculation. The letter (i or j) characterizes the influence of oil type and treatment. If it is different for the same oil for the two treatments, the influence of treatment is significantly different. If it is identical for the same treatment, the behaviour of both oils is not significantly different.)

treatment is significantly different. To show the influence of the oil type, P-value was compared to 0.05 (test at $95 \%$ confidence). In the heating process, the starting point (noted T0) is when oil reached $180^{\circ} \mathrm{C}$ and not the fresh oil at room temperature (noted FO). In beginning of oxidation, $\mathrm{PV}$ increases from 15 to $87 \mathrm{meq} \mathrm{O}_{2} \cdot \mathrm{kg}^{-1}$ for green type oil and from 20 to $85 \mathrm{meq} \mathrm{O}_{2} \cdot \mathrm{kg}^{-1}$ for black type oil before to decrease after less half hour of heating. After $210 \mathrm{~min} \mathrm{PV}$ is $37 \mathrm{meq} \mathrm{O}_{2} \cdot \mathrm{kg}^{-1}$ for green type oil and $29 \mathrm{meq}$ $\mathrm{O}_{2} \cdot \mathrm{kg}^{-1}$ for black type oil. The oil type seems to have any influence on the thermal process because both oils show similar evolution of PV according to the ANOVA test 
$(\mathrm{P}$-value $=0.98)$. When the oils are submitted to a photochemical treatment, $\mathrm{PV}$ increases linearly and similarly for both oils from 15 to $412 \mathrm{meq} \mathrm{O}_{2} \cdot \mathrm{kg}^{-1}$ for green type and from 21 to $419 \mathrm{meq} \mathrm{O}_{2} \cdot \mathrm{kg}^{-1}$ for black type (P-value $\left.=0.91\right)$. No decrease of this index occurs. For both treatments, after $30 \mathrm{~min}$, PV already exceeds the European regulation limit for EVOO [5]. The PV increase traduces the formation of peroxides and hydroperoxides (primary products of oxidation). Their transformation into secondary oxidation products like alcohols, acids, ketones or aldehydes [8], translates by PV decease, has not occurred during the photochemical treatment. Thus, as it increases in the early stage of oxidative treatment, PV is a good first step oxidation indicator. After, quantification of aldehydes through AV is necessary to quantify oxidation. AV increases during the first 90 minutes of heating, from 24 to 278 for green type oil and from 39 to 277 for black type oil, then decrease until 167 for both after 210 min of heating. Both oils have the same behaviour according to the ANOVA test on AV $(\mathrm{P}$-value $=0.72)$. The generation of aldehydes has occurred without induction time as soon as peroxides and hydroperoxides were formed showing their short time life. AV decrease may be explained by the transformation of long chain non volatile aldehydes into short chain esters or acids [35]. AV limit commonly used by different authors to characterize EVOO, i.e. 10 [13] [36], is quickly overtaken. Conversely, this degradation has not occurred when oils were photo-treated. Instead, a large induction time was observed prior to the formation of aldehydes from peroxides and hydroperoxides. AV rises up only after $90 \mathrm{~min}$ for black type (from 5 to 21) and $150 \mathrm{~min}$ for green type oil (from 8 to 18), and AV limit is overtaken almost starting from these times. The evolution of AV is similar for the two oil types $(\mathrm{P}$-value $=0.14)$ notwithstanding the time shift observed for the green type oil, and substantially lower (10 times) than during the heat aging. So, heat seems to more promote the decomposition of peroxides and hydroperoxides. For an accurate estimation of the oxidation stage, both parameters PV and AV should be simultaneously interpreted through TOTOX. For the thermal heating, it takes into account both the evolution of peroxides and aldehydes. This is reflected by a time at which a maximum is reached, shifted upwards compared to the PV curve, and, a maximum value is maintained over a longer period of time (within 60 $\min$ ) reflecting the formation of aldehydes and their importance in the oxidation process. For the photochemical ageing, as the contribution of aldehydes in the determination of TOTOX is weak, it is redundant with PV and it evolves in the same way. However, broadly, TOTOX is a better indicator of oxidation.

$\mathrm{FA}, \mathrm{K}_{232}$ and $\mathrm{K}_{270}$ indices evolution are respectively represented in Figures 2(a)-(c) for thermal-oxidation and during the photochemical treatment (Figures 2(d)-(f)) for both oils. Their evolutions during thermal ageing are similar for the both oil types. Indeed, the F-test for the equivalence of variances provides p-values greater than 0.05 (respectively $0.74,0.85$ and 0.23 ). FA is an important quality index used as a criterion for classifying olive oil in various commercial grades [5]. It increases from $1.0 \%$ to $3.7 \%$ for black type and from $0.3 \%$ to $3.6 \%$ for green type during 210 minutes, and exceed the European regulation limits $(0.8 \%$ for EVOO and $2 \%$ for VOO [5]) after 1 hour. Even if the value of FA of black oil is higher than the green oil one, according to ANOVA test, 

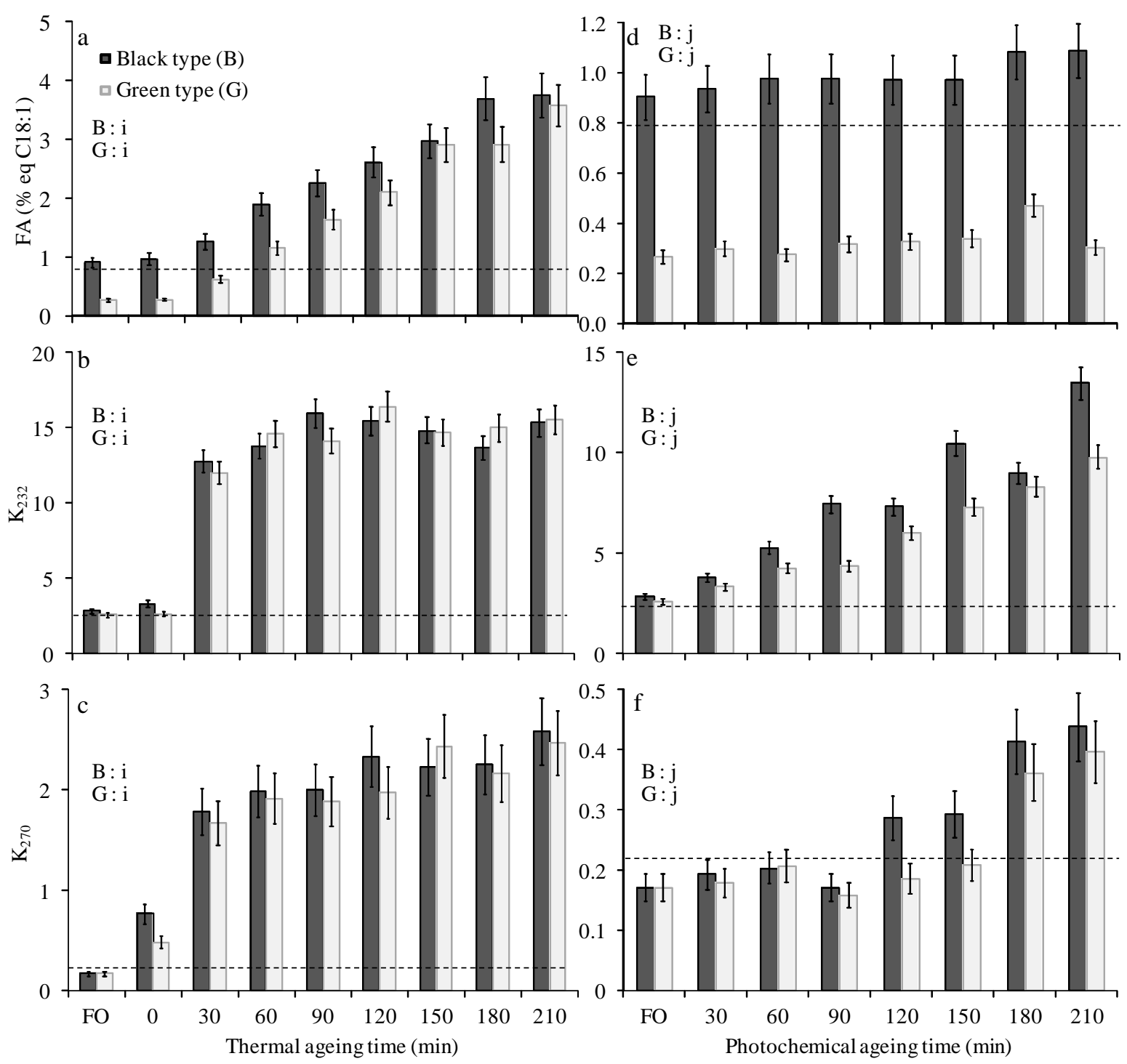

Figure 2. Evolution of (a, d) free acidity (FA), (b, e) $K_{232}$ and (c, f) $K_{270}$ according to thermal and photochemical oxidation time. (Limit fixed by the European regulation for EVOO (EEC, 2013); C18:1, oleic acid. Values are the means \pm standard deviation of 3 replicate analyses. The signification of letter ( $i$ or $j$ ) is the same than for Figure 1.)

FA has had the same linear increase for both oil types that reveals the accumulation of acids, the final oxidation products. In contrast, no evolution has been noticeable during the photochemical treatment. Indeed, the average deviation is smaller than the experimental error (respectively $4 \%$ and $3 \%$ versus $9 \%$ ).

$\mathrm{K}_{232}$ and $\mathrm{K}_{270}$, rise rapidly in the beginning of thermal oxidation from about 3 to 12.5 for $\mathrm{K}_{232}$ and from 0.5 to 1.7 for $\mathrm{K}_{270}$ in one half hour. Then, these parameters remain almost constant at about 15.5 and 2.5 and widely exceed the European regulation limits, respectively 2.5 and 0.2 for EVOO and 2.60 and 0.25 for VOO [5]. However, a decreasing should be expected because PUFA decrease during thermal oxidation [37]. Then, it seems that some degradation products, other than conjugated dienes and trienes, absorb at these wavelengths, which distort the measurement as shown particularly for $\mathrm{K}_{270}$ 
[38]. Therefore, these indices are not suitable for highly oxidized oils. When oils were photo-oxidized, $\mathrm{K}_{232}$ and $\mathrm{K}_{270}$ values increase slower. After $210 \mathrm{~min}$, even if the final values (upper to the European regulation limit) are higher for black type than for green type (13.5 versus 9.8 for $\mathrm{K}_{232}$ and 0.44 versus 0.40 for $\mathrm{K}_{270}$ ) ANOVA tests do not show significant difference of behaviour of the two oils ( $\mathrm{p}$-values greater than 0.05 , respectively 0.41 and 0.70 ).

\subsubsection{Fatty Acid Composition}

An important parameter to estimate the oxidative stability is the fatty acid composition of triglycerides and their unsaturation degree in particular. Green type oil and black type oil have initially similar composition because these oils come from olives of the same cultivar. Evolution of the contents of fatty acid of triglycerides over time is represented by unsaturation classes in Table 2 where ANOVA results are transcribed by letters as in Figure 1 and Figure 2. The ANOVA performed on the SFA, MUFA and PUFA data sets has shown no significant differences between green and black type oils whatever the ageing process: p-values are greater than 0.05 (respectively $0.71,0.85$ and 0.90 for the thermal ageing, and $0.65,0.82$ and 0.26 for photochemical ageing). During the thermal ageing, decreases of at least $17 \%, 44 \%$ and $85 \%$ were recorded for SFA, MUFA and PUFA respectively. However, fatty acids were less affected by photochemical treatment than by the thermal one. Indeed, P-values obtained when thermal and photochemical treatments were compared by ANOVA were lower than 0.05 (respectively $0.046,0.0001$ and 0.0002 ). The SFA are stable, the MUFA decrease of at least $11 \%$ and the PUFA fall to about 24\%. During both ageing, the deterioration of PUFA was much more intense. Indeed, unsaturations weaken carbon chains as explained by

Table 2. Evolution of saturated fatty acid (SFA), mono unsaturated fatty acid (MUFA) and poly unsaturated fatty acid (PUFA) contents expressed as $\mathrm{mg} \cdot \mathrm{eq}_{19} \cdot \mathrm{g}^{-1}$ according to thermal and photochemical oxidation time for green type oil (G) and black type oil (B).

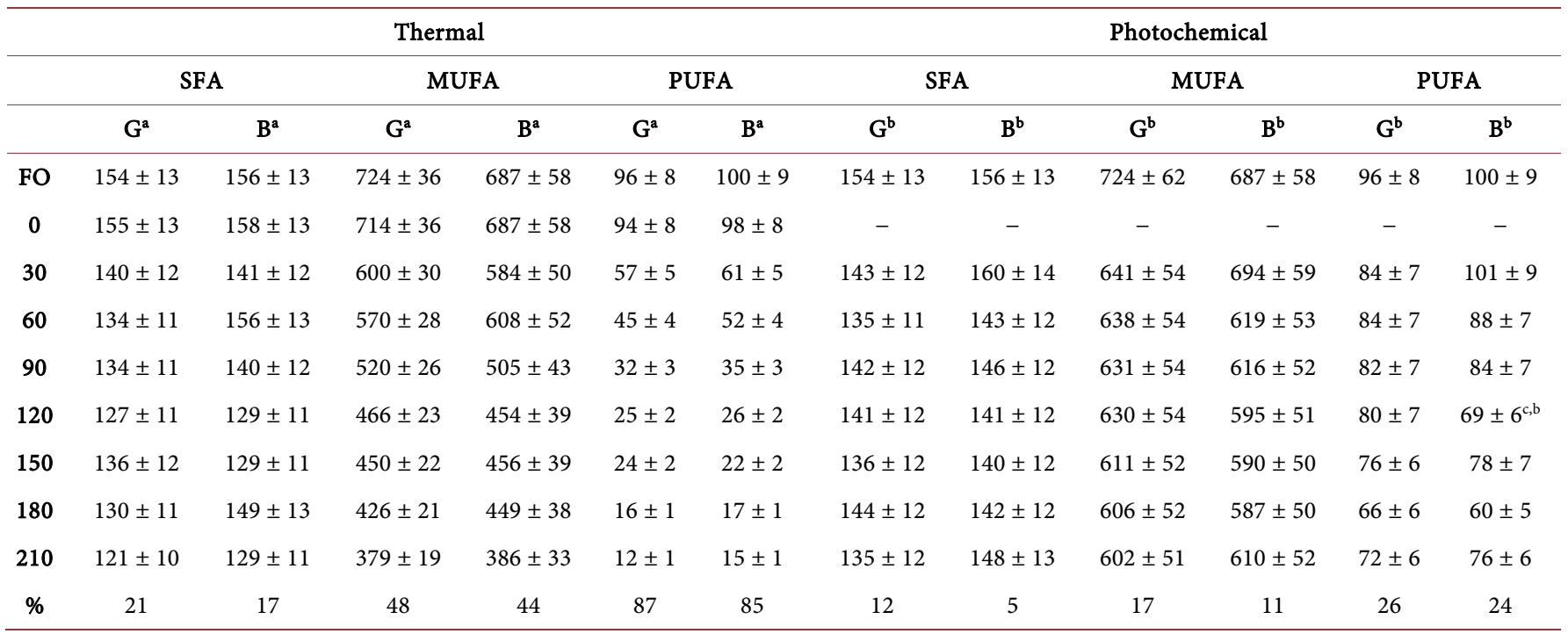

Values are the means \pm standard deviation of 3 replicate analyses. FO, fresh oil; \%, overall percentage of decrease. The letter (a or b) characterizes the influence of oil type and treatment. If it is identical for the same treatment, the behaviour of both oils is not significantly different. If it is identical for the same oil for the two treatments, the influence of treatment is not significantly different. 
Dobson et al. [39] and promote the abstraction of hydrogen adjacent to the double bond and the formation of free radicals [36].

\subsubsection{Phenolic Components}

Evolution of Tyr and HO-Tyr contents over thermal ageing time is represented in Figure 3 (a) and Figure 3(c) and over photochemical ageing time in Figure 3(b) and Figure 3(d) for each oil type. Tyr and HO-Tyr are the main products in the final stage of degradation of the two major secoiridoids in olive drupes, respectively oleuropein and ligstroside, which therefore are both present in oil as well as a number of their derivatives formed during olive oil extraction and later during storage of oil [21]. During thermal treatment of green type oil, Tyr has been highly degraded but still detected after $210 \mathrm{~min}$, while in black type oil, it has been more preserved. ANOVA gives a p-value lower than 0.05 ( $\mathrm{p}$-value $=0.01)$ showing that, for this compound, the two oils have had a different behaviour. So, green type oil seems to be more "resistant" than the black type oil. This trend was confirmed with the evolution of HO-Tyr, which has completely disappeared as early as the 10 min heating in the black type oil and after only half an hour in green type oil. These results are in accordance with previous literature and confirm the higher antioxidant power of HO-Tyr, which by its deterioration protects the triglycerides [14] [21] [22]. During the photochemical ageing, the Tyr
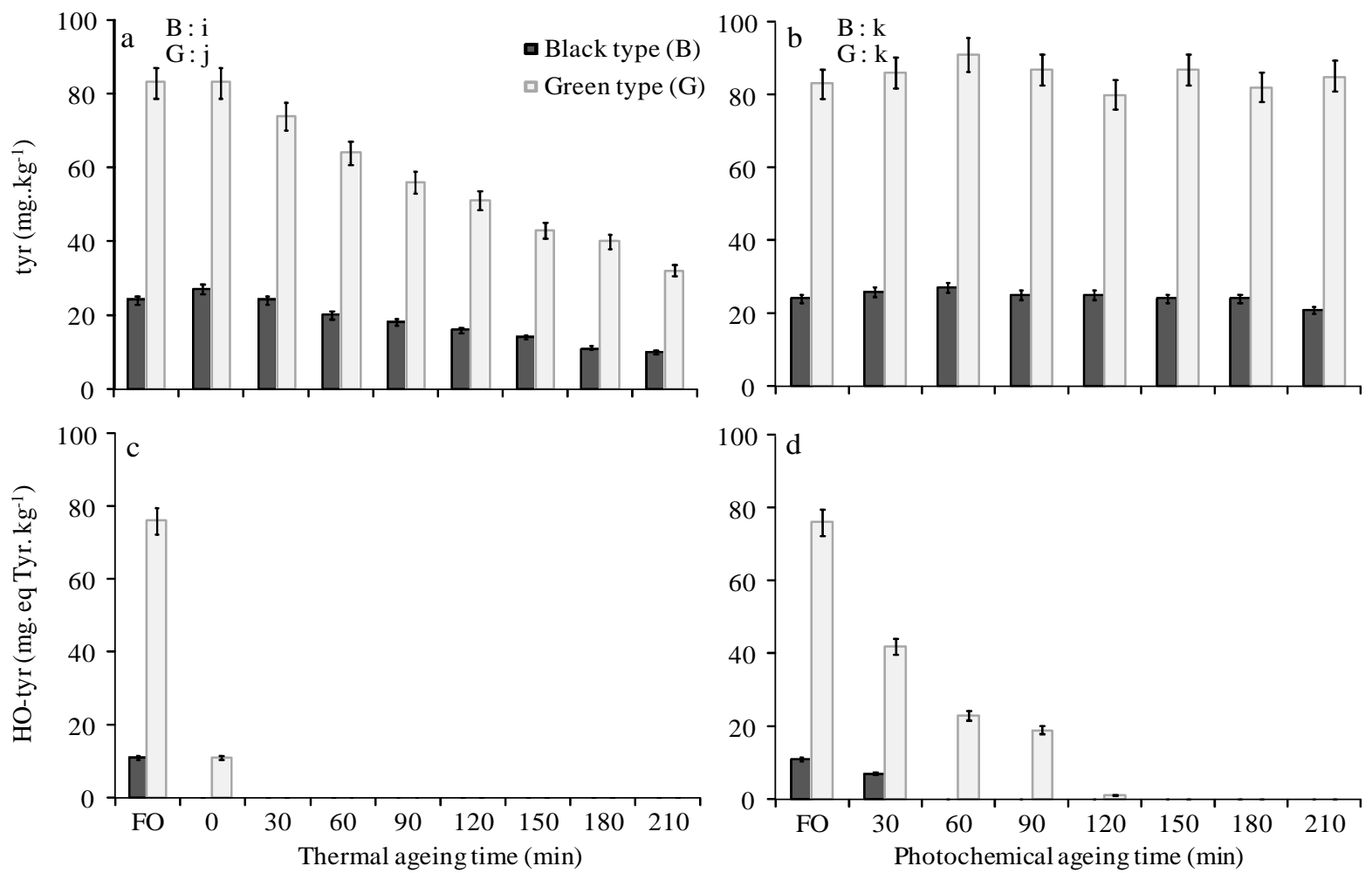

Figure 3. Evolution of (a, c) tyrosol content (Tyr) and (b, d) hydroxyl-tyrosol content (HO-Tyr) expressed as mg.eq Tyr. $\mathrm{kg}^{-1}$ according to thermal and photochemical oxidation time. (Values are the means \pm standard deviation of 3 replicate analyses. The letter ( $\mathrm{j}$ or $\mathrm{k}$ ) characterizes the influence of oil type and treatment. For almost samples, HO-Tyr is not detected (limit of detection $=0.1 \mathrm{mg} \cdot \mathrm{eq}$ Tyr $\cdot \mathrm{kg}^{-1}$ ), ANOVA analysis has no meaning.) 
content does not evolve over time. Indeed, the average deviation is close to the experimental error (about 5\%), and HO-Tyr has been slowly degraded than during thermal oxidation. The persistence of HO-Tyr for longer time (two hours), may explain the significant persistence of hydroperoxides (high PV value) which are not degraded into aldehydes or acids (low value of AV and FA). Indeed, antioxidants as HO-Tyr or Tyr act by reacting with lipid radicals [22], which would no longer be operative in the termination phase of radical chain reactions.

\subsubsection{Principal Component Analysis}

To compare the oils behaviour according the ageing processes, the oils oxidative stability and to determine the significant indices of oils quality, a principal component analyse (PCA) has been performed on all aged samples with all indices as variables (Figure 4). The two first components explain $78 \%$ of the total variance (respectively $56 \%$ and $22 \%$ for PC1 and PC2). The score scatter plot (Figure 4(a)) shows the similarities of green and black type samples. The first component PC1 separates samples in two groups, no-oxidized samples and photo-oxidized samples in the positive part and thermal oxidized samples in the negative part according to oxidation time. The second component PC2 separates samples according to the photochemical oxidation time. The impact of the heating and the light on the chemical quality parameters is significant after 30 minutes because the samples are positioned according to the oxidation time. Loadings plot (Figure $4(\mathrm{~b})$ ) shows that FA, AV, $\mathrm{K}_{232}$ and $\mathrm{K}_{270}$, located in the PC1 negative side, are correlated to the thermal oxidized samples. MUFA, PUFA, SFA and HO-Tyr, located in the bottom write quarter, are the best indicators for non-oxidized oils. The last photo-oxidation times (after $90 \mathrm{~min}$ ) are characterised by high values of PV and TOTOX. Green and black type oils have a same behaviour when thermal oxidized, since the distribution of samples according to the oxidation times (Figure 4(a)) is almost the same regardless of the type of oil. Concerning the photo-oxidized samples, black type samples are shifted to the left compared with green type samples for the same time. Black type oils separated from the green type oils, according to PC3 (10\% of the total variance explained, Figure 4(c)) are characterised by SFA and FA (Figure 4(d)) while Tyr and HO-Tyr characterise the green type oils. This analyse confirms the previous results obtains by ANOVA on indices.

\section{Conclusion}

Oxidative stability of two virgin olive oils (green type and black type) has been studied during thermal and photochemical treatments through the evolution of legal quality indices. No differences have been detected for green and black type oils when samples have been heated: black type oil is not worse than green type oil. Nevertheless, green type oil has shown better oxidative stability upon the UV treatment because of its higher phenolic content. Moreover, the heating has more impacted the oil composition than the UV irradiations. The thermal treatment has induced a rapid formation of peroxides and aldehydes degradation of which has lead to great linear increase of FA. An almost complete disappearance of PUFA and a great loss of MUFA were recorded after 

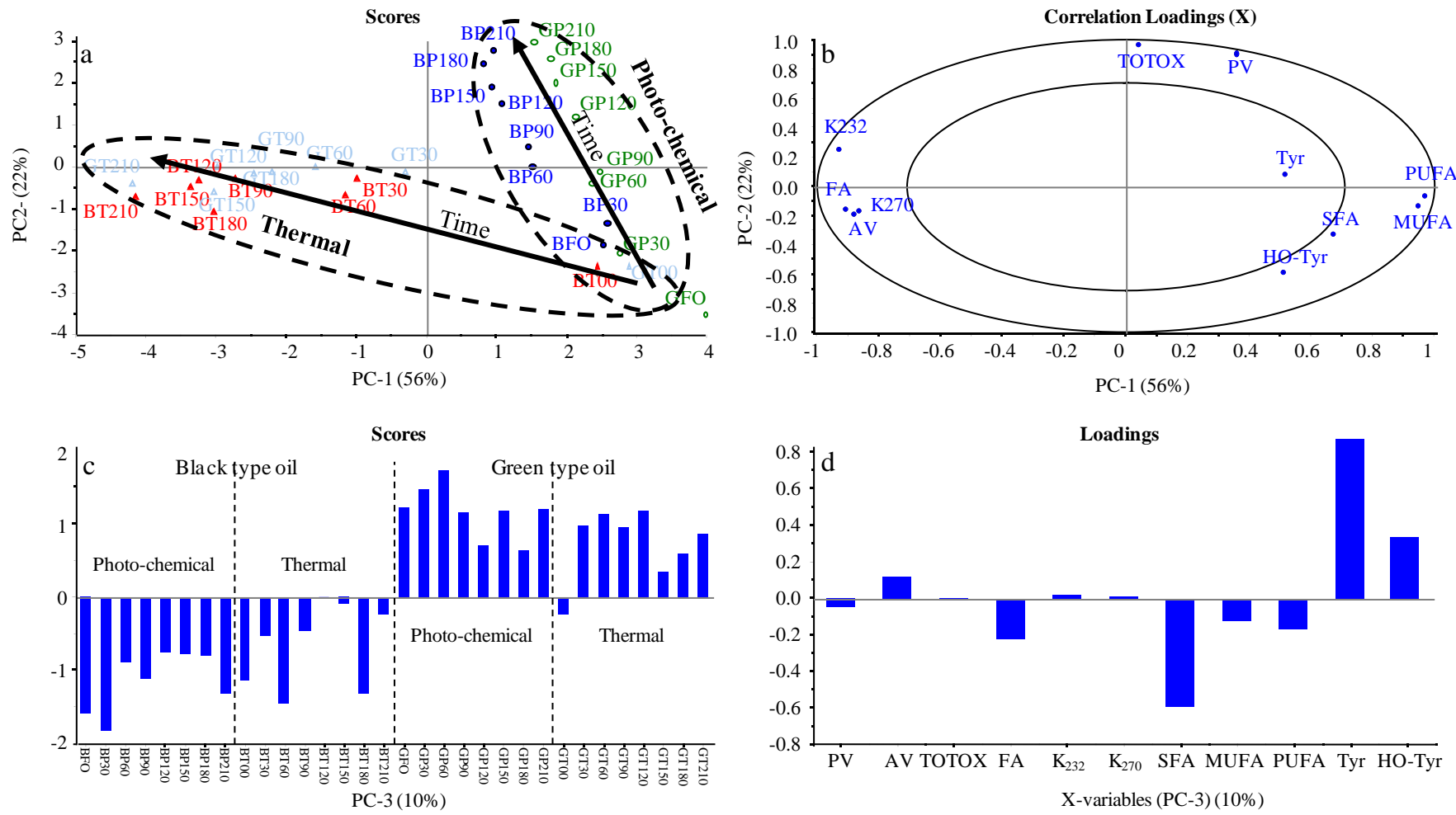

Figure 4. (a, c) Score and (b, d) loading plots of PCA performed on chemical parameters obtained after thermal and photochemical treatments. (PC, principal component; PV, peroxide value; AV, p-anisidine value; TOTOX, total oxidation value; FA, free acidity; $\mathrm{K}_{232}$, spectroscopic absorption at $232 \mathrm{~nm} ; \mathrm{K}_{270}$, spectroscopic absorption at $270 \mathrm{~nm}$; SFA, saturated fatty acids; MUFA, mono unsaturated fatty acids; PUFA, poly unsaturated fatty acids; Tyr, tyrosol; HO-Tyr, hydroxyl-tyrosol.)

210 min. During the photochemical treatment, a greater quantity of peroxides and few aldehydic compounds have been formed. The evolution of PUFA and MUFA were less pronounced. Only FA value of the green type oil remained below the European regulation limit after the UV irradiation. PCA analysis, has demonstrated that the best indicators for characterizing non-oxidized oils are MUFA, PUFA and SFA, FA and AV for control of thermal oxidation and PV and TOTOX for photo-oxidation control. Therefore, from a practical standpoint, these results support the fact that precautions must be taken when cooking to avoid high temperatures for a long time, because different components from degradation can change the taste of some food [40] and/or be harmful [41]. Even if the degradation is lower during the UV irradiation, it's better to keep oil away from light.

\section{Acknowledgements}

Authors want to thank Pr Jacques Artaud for his advice and knowledge and Noelly Thomas and Sabrina Abdi for their technical support.

\section{References}

[1] ISO (2009) Animal and Vegetable Fats and Oils-Determination of Acid Value and Acidity. ISO Standard 660, International Organization for Standardization Geneva, Switzerland. 
[2] ISO (2007) Animal and Vegetable Fats and Oils-Determination of Peroxide Value-Iodometric (Visual) Endpoint Determination. ISO Standard 3960, International Organization for Standardization Geneva, Switzerland.

[3] International Olive Council (2010) Spectrophotometric Investigation in the Ultraviolet Adopted by International Olive Council. IOC/T.20/Doc. No.19.

[4] ISO (2006) Animal and Vegetable Fats and Oils-Determination of Anisidine Value. ISO Standard 6885, International Organization for Standardization Geneva, Switzerland.

[5] EEC (2013) On the Characteristics of Olive Oil and Olive-Residue Oil and on the Relevant Methods of Analysis. Commission Implementing Regulation (EU) 299/2013 of March 26, 2013 Amending Regulation EEC 2568/91. Official Journal of the European Communities, 52-70.

[6] International Olive Council (2013) Preparation of the Fatty Acid Methyl Esters from Olive Oil and Olive-Pomace Oil Adopted by International Olive Council. IOC/T.20/Doc. No. 24.

[7] International Olive Council (2009) Determination of Biophenols in Olive Oils by HPL Cadopted by International Olive Council. IOC/T.20/Doc. No. 29.

[8] Gertz, C., Klostermann, S. and Kochhar, S.P. (2000) Testing and Comparing Oxidative Stability of Vegetable Oils and Fats at Frying Temperature. European Journal of Lipid Science and Technology, 102, 543-551.

[9] Niccolo, M.B.C., Neri, M.B. and Sebastiano, M.B. (2000) Method for Preservation of Olive Oil. Patent EP0974270A1.

[10] Intelidimou, A.D. (2014) Olive Oil Portions. Patent GR1008762B.

[11] Santos, S.P., Cruz, R., Cunha, S.C. and Casal, S. (2013) Effect of Cooking on Olive Oil Quality Attributes. Food Research International, 54, 2016-2024.

https://doi.org/10.1016/j.foodres.2013.04.014

[12] Andrikopoulos, N.K., Kalogeropoulos, N., Falirea, A. and Barbagianni, M.N. (2002) Performance of Virgin Olive Oil and Vegetable Shortening during Domestic Deep-Frying and Pan-Frying of Potatoes. International Journal of Food Science Technology, 37, 177-190. https://doi.org/10.1046/j.1365-2621.2002.00555.x

[13] Casal, S., Malheiro, R., Sendas, A., Oliveira, B.P.P. and Pereira, J.A. (2010) Olive Oil Stability under Deep-Frying Conditions. Food and Chemical Toxicology, 48, 2972-2979. https://doi.org/10.1016/j.fct.2010.07.036

[14] Cheikhousman, R., Zude, M., Bouveresse, D., Léger, C., Rutledge, D. and Birlouez-Aragon, I. (2005) Fluorescence Spectroscopy for Monitoring Deterioration of Extra Virgin Olive Oil during Heating. Analytical and Bioanalytical Chemistry, 382, 1438-1443. https://doi.org/10.1007/s00216-005-3286-1

[15] Daskalaki, D., Kefi, G., Kotsiou, K. and Tasioula-Margari, M. (2009) Evaluation of Phenolic Compounds Degradation in Virgin Olive Oil during Storage and Heating. Journal of Food and Nutrition Research, 48, 31-41.

[16] Mancebo-Campos, V., Desamparados-Salvador, M. and Fregapane, G. (2007) Comparative Study of Virgin Olive Oil Behavior under Rancimat Accelerated Oxidation Conditions and Long-Term Room Temperature Storage. Journal of Agricultural and Food Chemistry, 55, 8231-8236. https://doi.org/10.1021/jf070915y

[17] Maggio, R.M., Valli, E., Bendini, A., Gómez-Caravaca, A.M., Toschi, T.G. and Cerretani, L. (2011) A Spectroscopic and Chemometric Study of Virgin Olive Oils Subjected to Thermal Stress. Food Chemistry, 127, 216-221. https://doi.org/10.1016/j.foodchem.2010.12.018

[18] Gharby, S., Harhar, H., Matthäus, B., Bouzoubaa, Z. and Charrouf, Z. (2016) The Chemical 
Parameters and Oxidative Resistance to Heat Treatment of Refined and Extra Virgin Moroccan Picholine Olive Oil. Journal of Taibah University for Science, 10, 100-106. https://doi.org/10.1016/j.jtusci.2015.05.004

[19] Poyato, C., Ansorena, D., Navarro-Blasco, I. and Astiasarán, I. (2014) A Novel Approach to Monitor the Oxidation Process of Different Types of Heated Oils by Using Chemometric Tools. Food Research International, 57, 152-161. https://doi.org/10.1016/j.foodres.2014.01.033

[20] Gómez-Alonso, S., Mancebo-Campos, V., Salvador, M.D. and Fregapane, G. (2007) Evolution of Major and Minor Components and Oxidation Indices of Virgin Olive Oil during 21 Months Storage at Room Temperature. Food Chemistry, 100, 36-42. https://doi.org/10.1016/j.foodchem.2005.09.006

[21] Bešter, E., Butinar, B., Bučar-Miklavčič, M. and Golob, T. (2008) Chemical Changes in Extra Virgin Olive Oils from Slovenian Istra after Thermal Treatment. Food Chemistry, 108, 446-454. https://doi.org/10.1016/j.foodchem.2007.10.061

[22] Gómez-Alonso, S., Fregapane, G., Salvador, M.D. and Gordon, M.H. (2003) Changes in Phenolic Composition and Antioxidant Activity of Virgin Olive Oil during Frying. Journal of Agricultural and Food Chemistry, 51, 667-672. https://doi.org/10.1021/jf025932w

[23] Škevin, D., Rade, D., Štrucelj, D., Mokrovčak, Ž., Neđeral, S. and Benčić, Đ. (2003) The Influence of Variety and Harvest Time on the Bitterness and Phenolic Compounds of Olive Oil. European Journal of Lipid Science and Technology, 105, 536-541.

[24] Poulli, K.I., Mousdis, G.A. and Georgiou, C.A. (2009) Monitoring Olive Oil Oxidation under Thermal and UV Stress through Synchronous Fluorescence Spectroscopy and Classical Assays. Food Chemistry, 117, 499-503. https://doi.org/10.1016/j.foodchem.2009.04.024

[25] Rahmani, M. and Csallany, A.S. (1998) Role of Minor Constituents in the Photooxidation of Virgin Olive Oil. Journal of the American Oil Chemists' Society, 75, 837-843. https://doi.org/10.1007/s11746-998-0234-1

[26] Rodrigues, N., Dias, L.G., Veloso, A.C.A., Pereira, J.A. and Peres, A.M. (2016) Monitoring Olive Oils Quality and Oxidative Resistance during Storage Using an Electronic Tongue. LWT_Food Science and Technology, 73, 683-692. https://doi.org/10.1016/j.lwt.2016.07.002

[27] Luna, G., Morales, M.T. and Aparicio, R. (2006) Changes Induced by UV Radiation during Virgin Olive Oil Storage. Journal of Agriculture and Food Chemistry, 54, 4790-4794. https://doi.org/10.1021/jf0529262

[28] Nieto, M.L., Hodaifa, G., Rodriguez Vives, S., Gimenez Casares, J.A. and Casanova, M.S. (2009) Photodegradation of Phytosanitary Molecules Present in Virgin Olive Oil. Journal of Photochemistry and Photobiology A: Chemistry, 203, 1-6. https://doi.org/10.1016/j.jphotochem.2008.11.025

[29] Kotsiou, K. and Tasioula-Margari, M. (2016) Monitoring the Phenolic Compounds of Greek Extra-Virgin Olive Oils during Storage. Food Chemistry, 200, 255-262. https://doi.org/10.1016/j.foodchem.2015.12.090

[30] Pinatel, C., Petit, C., Ollivier, D. and Artaud, J. (2004) Outils pour l'amélioration organoleptique des huiles d'olives vierges. OCL_Oilseeds \& Fats Corps and Lipids, 11, 217-222. https://doi.org/10.1051/ocl.2004.0217

[31] Afidol, Association Française interprofessionnelle de l'olive (2013). http://www.afidoltek.org/index.php/Fruit\%C3\%A9 noir

[32] Talpur, M.Y., Sherazi, S.T.H., Mahesar, S.A. and Bhutto, A.A. (2010) A Simplified UV Spectrometric Method for Determination of Peroxide Value in Thermally Oxidized Canola 
Oil. Talanta, 80, 1823-1826. https://doi.org/10.1016/j.talanta.2009.10.028

[33] Nissiotis, M. and Tasioula-Margari, M. (2002) Changes in Antioxidant Concentration of Virgin Olive Oil during Thermal Oxidation. Food Chemistry, 77, 371-376. https://doi.org/10.1016/S0308-8146(02)00113-9

[34] Jolliffe, I.T. (2002) Principal Component Analysis. 2nd Edition, Springer, Berlin.

[35] Frankel, E. (1984) Lipid Oxidation: Mechanisms, Products and Biological Significance. Journal of the American Oil Chemists' Society, 61, 1908-1917. https://doi.org/10.1007/BF02540830

[36] Naz, S., Siddiqi, R., Sheikh, H. and Sayeed, S.A. (2005) Deterioration of Olive, Corn and Soybean Oils due to Air, Light, Heat and Deep-Frying. Food Research International, 38, 127-134. https://doi.org/10.1016/j.foodres.2004.08.002

[37] Moreno, M.M.C.M., Olivares, M.D., López, A.F.J., Adelantado, G.J.V. and Reig, B.F. (1999) Analytical Evaluation of Polyunsaturated Fatty Acids Degradation during Thermal Oxidation of Edible Oils by Fourier Transform Infrared Spectroscopy. Talanta, 50, 269-275. https://doi.org/10.1016/S0039-9140(99)00034-X

[38] Shahidi, F. and Wanasundara, U.N. (2002) Methods for Measuring Oxidative Rancidity in Fats and Oils. In: Akoh, C.C. and Min, D.B., Eds., Food Lipids, Chemistry, Nutrition, and Biotechnology, Marcel Dekker Inc., New York, 465-505. https://doi.org/10.1201/9780203908815.ch14

[39] Dobson, G., Christie, W.W. and Dobarganes, M.C. (1996) Changes in Molecular Species of Triacylglycerols during Frying. Consejo Superior de Investigaciones Cientificas (CSIC), 47, 34-37.

[40] Koh, E. and Surh, J. (2015) Food Types and Frying Frequency Affect the Lipid Oxidation of Deep Frying Oil for the Preparation of School Meals in Korea. Food Chemistry, 174, 467472. https://doi.org/10.1016/j.foodchem.2014.11.087

[41] Sacchi, R., Paduano, A., Savarese, M., Vitaglione, P. and Fogliano, V. (2014) Extra Virgin Olive Oil: From Composition to "Molecular Gastronomy". Advances in Nutrition and Cancer, 159, 325-338. https://doi.org/10.1007/978-3-642-38007-5 19

\section{Abbreviations}

ANOVA, analysis of variance; C16:0, palmitic acid (hexadecanoic acid); C16:1w9, hypogeic acid (7-hexadecenoic acid); C16:1 $\omega 7$, palmitoleic acid (9-hexadecenoic acid); C17:0, margaric acid (heptadecanoic acid); C17:1 $\mathbf{8} 8$, margaroleic acid (9-heptadecenoic acid);

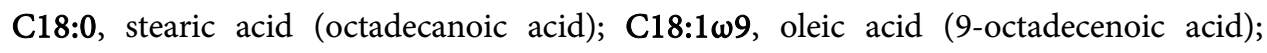

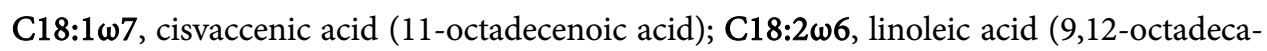
dienoic acid); C18:3 $\omega 3$, linolenic acid (9,12,15-octadecatrienoic acid); C19, nonadecanoate methyl ester; C20:0, arachidic acid (eicosanoic acid); C20:1 $\omega 9$, gondoic acid (11-eicosenoic acid); C22:0, behenic acid (docosanoic acid); C24:0, lignoceric acid (tetracosanoic acid); AV, p-anisidine value; FA, free acidity; FAME, fatty acid methyl esters; HO-Tyr, hydroxyl-tyrosol; IOC, International olive council; ISO, international standardisation organisation; K232, spectroscopic absorption at $232 \mathrm{~nm}$; K270, spectroscopic absorption at $270 \mathrm{~nm}$; MUFA, monounsaturated fatty acids; PCA, Principal component analysis; PUFA, polyunsaturated fatty acids; PV, peroxide value; SFA, saturated fatty acids; TOTOX, total oxidation value; Tyr, tyrosol; UV, ultra violet. 
Submit or recommend next manuscript to SCIRP and we will provide best service for you:

Accepting pre-submission inquiries through Email, Facebook, LinkedIn, Twitter, etc. A wide selection of journals (inclusive of 9 subjects, more than 200 journals)

Providing 24-hour high-quality service

User-friendly online submission system

Fair and swift peer-review system

Efficient typesetting and proofreading procedure

Display of the result of downloads and visits, as well as the number of cited articles

Maximum dissemination of your research work

Submit your manuscript at: http://papersubmission.scirp.org/

Or contact ajac@scirp.org 